\title{
Vorbemerkung zur 10. Auflage
}

Vor 25 Jahren ist „Management in der Hotellerie und Gastronomie“ erstmals erschienen. Seit dieser Zeit haben sich in der Branche weitreichende Veränderungen ergeben. Der Wettbewerb und die Konzentration haben zugenommen, die Betriebs- und die Personalkosten sind deutlich gestiegen. Mit der Corona-Pandemie ist, nach mehreren Jahren des Wachstums, kurz vor dem Druck dieser Auflage, eine in ihren Auswirkungen nicht erwartete neue Herausforderung aufgetreten. Mit umfassenden Konsequenzen, bis hin zur Existenzgefährdung vieler Betriebe. Die Anforderungen an die Branche und ihre Verbände haben damit weiter zugenommen, staatliche Unterstützungen sind unabdingbar geworden.

All diese Entwicklungen haben die Bedeutung betriebswirtschaftlicher Fragestellungen in der Branche weiter erhöht. Hierzu einen Beitrag zu leisten war und ist das Ziel dieses Buches: Es werden übergreifend Grundlagen für das erfolgreiche Management von Hotel- und Gaststättenbetrieben dargestellt. Über alle Jahre waren umfangreiche Aktualisierungen und Erweiterungen notwendig, auch bei dieser neuen Auflage. Aufgrund der zunehmenden Bedeutung wurde aktuell ein einführendes Kapitel Digitalisierung aufgenommen.

Im Kreis der Autorinnen und Autoren hat es immer wieder Veränderungen gegeben. Neue sind hinzugekommen, andere ausgeschieden. Mit großer Betroffenheit mussten wir kurz vor Drucklegung die Nachricht vom Tode von Frau Kay Winter und Herrn Axel Schrand zur Kenntnis nehmen. Beide haben Wichtiges für die Branche geleistet. Kay Winter als Autorin und Personaldirektorin namhafter Hotelgesellschaften, Axel Schrand als Autor und Dozent für Tourismuslehre und -marketing an verschiedenen Hochschulen.

Erfreulich ist, dass sich die Literaturlage in den vergangenen 25 Jahren erheblich erweitert hat. Für viele Fragestellungen, die im Buch angesprochen werden, stehen zwischenzeitlich vertiefende und weiterführende Veröffentlichungen zur Verfügung. Diese in früheren Jahren erhoffte, in diesem Maße kaum erwartete Entwicklung, ist als großer Fortschritt für die Hotellerie und Gastronomie zu sehen.

Danken möchte ich den Autorinnen und Autoren sowie allen, die über viele Jahre an der Fertigstellung dieses Buches mitgewirkt haben: Frau Uta Schlagenhauf vom Hotel Bareiss in Baiersbronn, Frau Geschäftsführerin Dunja Schlamminger vom DEHOGA Baden-Württemberg, Frau Katharina Sautter, Sonja Berlinger, Nadine Huber und Herrn Arndt Moritz Jaeschke, wissenschaftliche Mitarbeiterinnen am Steinbeis-Transferzentrum Tourismus und Hotellerie und der IWT Wirtschaft und Technik GmbH für die Überarbeitung der Texte. Vielen Dank auch dem Verlag DE GRUYTER Oldenbourg für die immer sehr angenehme und vertrauensvolle Zusammenarbeit und Partnerschaft.

Karl Heinz Hänssler 
